\title{
PhotoExcitation Dynamics in Electrochemically Charged CdSe Quantum Dots From Hot Carrier Cooling to Auger Recombination of Negative Trions
}

Honarfar, Alireza; Mourad, Hassan; Lin, Weihua; Polukeev, Alexey; Rahaman, Ahibur; Abdellah, Mohamed; Chábera, Pavel; Pankratova, Galina; Gorton, Lo; Zheng, Kaibo

Total number of authors:

11

Published in:

Applied Energy Materials

Link to article, DOI:

10.1021/acsaem.0c02478

Publication date:

2020

Document Version

Publisher's PDF, also known as Version of record

Link back to DTU Orbit

Citation (APA):

Honarfar, A., Mourad, H., Lin, W., Polukeev, A., Rahaman, A., Abdellah, M., Chábera, P., Pankratova, G., Gorton, L., Zheng, K., \& Pullerits, T. (2020). PhotoExcitation Dynamics in Electrochemically Charged CdSe Quantum Dots: From Hot Carrier Cooling to Auger Recombination of Negative Trions. Applied Energy Materials, 3(12), 12525-12531. https://doi.org/10.1021/acsaem.0c02478

\section{General rights}

Copyright and moral rights for the publications made accessible in the public portal are retained by the authors and/or other copyright owners and it is a condition of accessing publications that users recognise and abide by the legal requirements associated with these rights.

- Users may download and print one copy of any publication from the public portal for the purpose of private study or research.

- You may not further distribute the material or use it for any profit-making activity or commercial gain

- You may freely distribute the URL identifying the publication in the public portal 


\title{
Photoexcitation Dynamics in Electrochemically Charged CdSe Quantum Dots: From Hot Carrier Cooling to Auger Recombination of Negative Trions
}

\author{
Alireza Honarfar, Hassan Mourad, Weihua Lin, Alexey Polukeev, Ahibur Rahaman, Mohamed Abdellah, \\ Pavel Chábera, Galina Pankratova, Lo Gorton, Kaibo Zheng, and Tönu Pullerits*
}

Cite This: https://dx.doi.org/10.1021/acsaem.0c02478

Read Online

ABSTRACT: Fulfilling the potential of colloidal semiconductor quantum dots (QDs) in electrically driven applications remains a challenge largely since operation of such devices involves charged QDs with drastically different photophysical properties compared to their well-studied neutral counterparts. In this work, the full picture of excited state dynamics in charged CdSe QDs at various time scales has been revealed via transient absorption spectroscopy combined with electrochemistry as a direct manipulation tool to control the negative charging of CdSe QDs. In trions, excited states of single charged QDs, the additional electron in the conduction band speeds up the hot electron cooling by enhanced electron-electron scattering followed by charge redistribution and polaron formation in a picosecond time scale. The trions are finally decayed by the Auger process in a 500 ps time scale. Double charging in QDs, on the other hand, decelerates the polaron formation process while accelerates the following Auger decay. Our work demonstrates the potential of photoelectrochemistry as a platform for ultrafast spectroscopy of

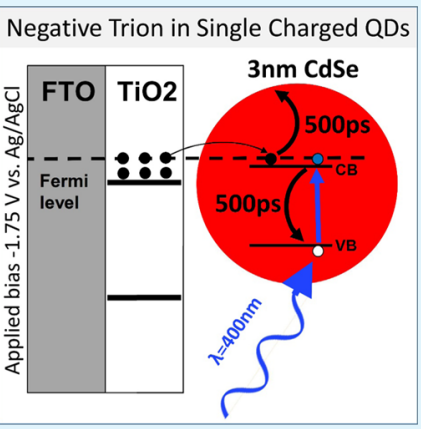
charged species and paves the way for further studies to develop comprehensive knowledge of the photophysical processes in charged QDs more than the well-known Auger decay, facilitating their use in future optoelectronic applications.

KEYWORDS: single charged, double charged, semiconductor nanocrystals, spectroelectrochemistry, transient absorption spectroscopy, polaron, tetron, Auger decay, solar cells, optoelectronics

\section{INTRODUCTION}

Quantum dots (QDs), ${ }^{1}$ as semiconductor nanocrystals with a size smaller than Bohr's radius, have been widely investigated both as model systems for fundamental research and for numerous applications. ${ }^{2-6}$ The applications typically rely on separation and extraction of photogenerated electron-hole pairs for the efficient conversion of photons to free charge carriers. ${ }^{7}$ This can be achieved through transfer of photogenerated charges to electron (hole) acceptors, which highly depends on the relative position of the band edges. ${ }^{8}$ Charge dynamics in such systems have been extensively investigated by time-resolved spectroscopies. ${ }^{7-9}$ Most of these studies are implemented on half-cell systems (i.e., only photoanodes or photocathodes) or under open-circuit conditions where electron/hole extraction through external circuits does not occur. In full functioning devices, extraction of charges to/from the electrodes is essential for device operation, which means that under working conditions, the photoactive layer can accumulate charges due to defects or Schottky barrier formation at the interfaces. ${ }^{10}$ Charge accumulation at the interfaces and also in the QDs considerably influences the device performance and optoelectronic behavior. ${ }^{11-14}$ Therefore, understanding of the effect of an external bias and the presence of extra charges in the QD-acceptor system is of great importance for the development of high-performance QD devices.

By absorption of light in a semiconductor, an electron-hole pair, exciton, is created. Two excitons in a QD interact and form a biexciton, which rapidly decays through the Auger process leaving behind a single exciton. ${ }^{15}$ Auger recombination in QDs is efficient ${ }^{16}$ and drastically influences the performance of the corresponding devices at high excitation intensities. Photoexcitation of a charged QD leads to the so-called trion, which is a three-body state of an electron-hole pair and an additional charge. Depending on the charge, a trion can be either positive or negative. Analogously to the biexciton, the trion also can decay through Auger recombination. ${ }^{15}$ The depopulation of trions through the Auger process competes with the extraction of charges and consequently undermines the charge separation efficiency in the devices. Most of the existing studies on trion dynamics utilized time-resolved

Received: October 7, 2020

Accepted: November 17, 2020 
photoluminescence spectroscopy to measure radiative trion decay in core/shell heterojunction structures ${ }^{15,17}$ where the shell structure has a direct effect on the trion recombination. ${ }^{18}$ Such a core-shell structure isolates the QD core from surface defects, and at the same time, the shell also slows down or even prevents charge carrier transport to and from the core, making integrated optoelectronics based on QDs difficult. ${ }^{19}$

In this work, we combine electrochemistry with ultrafast transient absorption spectroscopy (TA) to monitor changes in the excited state dynamics of the $\mathrm{QD}$ under controlled charging. ${ }^{12,20-23}$ By observing the changes in the steady state absorption during spectroelectrochemistry measurement, we have identified distinct potentials that correspond to the injection of one and two electrons to the QDs. The presence of these negative charges leads to negative trions and tetrons upon excitation by laser light in the TA measurement. ${ }^{15} \mathrm{We}$ observed a series of changes in the relaxation processes due to the extra electrons including nonradiative Auger recombination in a 500 ps time scale. We anticipate that our findings will pave the way to comprehensive knowledge and better understanding of the photophysical processes in charged QDs, leading to their efficient use in future nanotechnology applications.

\section{ELECTROCHEMISTRY}

In electrochemistry, the applied potential induces electron exchange between working electrodes and the sample under study. ${ }^{24}$ By changing the potential of the working electrode linearly with time (vs a reference electrode) from one set initial potential to another preset chosen potential and then reversing the scan linearly back to the initial potential while measuring the current that passes to the counter electrode, a cyclic voltammogram $(\mathrm{CV})$ is obtained, ${ }^{25}$ showing distinct bands at the potentials where charge carriers can enter the electronic states of the system. ${ }^{14,24,26}$ Monodispersed CdSe QDs with a diameter of $\sim 3 \mathrm{~nm}$ were used for sensitization of $\mathrm{TiO}_{2}$-coated fluorine-doped tin oxide (FTO) (Supporting Information). Such a $\mathrm{TiO}_{2}$-FTO system is analogous to the photoanode architecture of QD-sensitized solar cells. ${ }^{7}$ CVs of CdSe QDs were measured in a conventional three-electrode electrochemical cell configuration, where the $\mathrm{QD}-\mathrm{TiO}_{2}-\mathrm{FTO}$ assembly serves as the working electrode, a leakless pseudo$\mathrm{Ag} \mid \mathrm{AgCl}$ electrode is used as reference, and a platinum wire acts as a counter electrode. The detailed description is given in the Supporting Information. All of the potentials in this work are reported vs the $\mathrm{Ag} \mid \mathrm{AgCl}$ pseudo-reference. In equilibrium, the Fermi levels of the semiconductors that are in contact with each other are considered to be equal, which will equilibrate the charges through $\mathrm{QD}-\mathrm{TiO}_{2}$-FTO layers. ${ }^{27}$ In that respect, the $\mathrm{TiO}_{2}$-FTO layer can be considered to be as one system with a common Fermi level, while CdSe QDs are separate semiconductors attached to the $\mathrm{TiO}_{2}$-FTO layer by a linker molecule. The reference electrode is considered to have a fixed potential, and thus applying a negative bias corresponds to an increase in the $\mathrm{TiO}_{2}$-FTO Fermi level. At sufficiently high negative potentials, electrons in the conduction band $(\mathrm{CB})$ of the $\mathrm{TiO}_{2}$-FTO layer can reach energies equal to or higher than those of the CdSe CB; hence, electrons can be injected into the QDs. When electrons are exchanged between QDs and $\mathrm{TiO}_{2}$-FTO, a current change can be detected in the CV, which is represented in Figure 1a. For a better understanding of the electrochemical measurements and changes in the current, it is essential to define the relation between energy levels of the

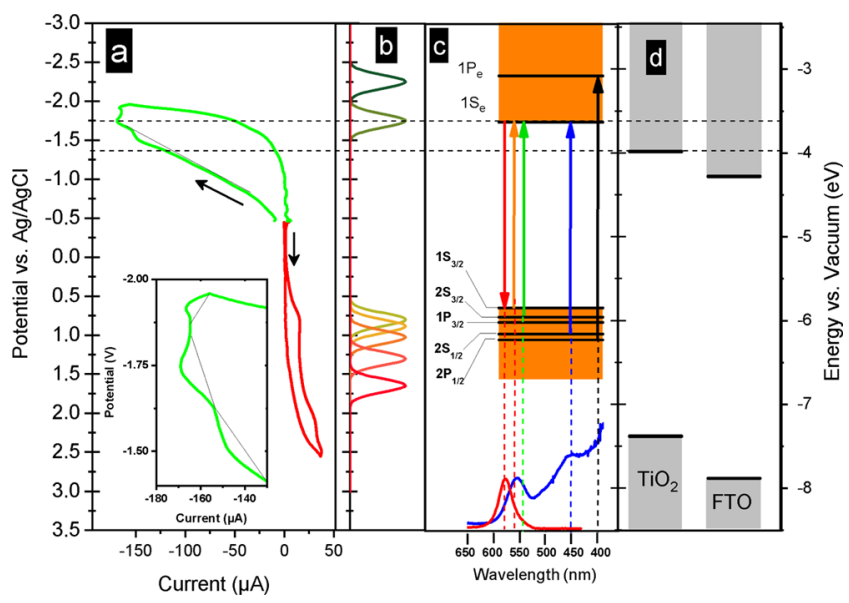

Figure 1. (a) Cyclic voltammogram of the $3 \mathrm{~nm} \mathrm{CdSe} \mathrm{QD} / \mathrm{TiO}_{2} /$ FTO electrode assembly. Measurement was performed in a threeelectrode cell where the CdSe QD assembly on a $\mathrm{TiO}_{2}$-coated FTO electrode served as the working electrode, a pseudo $\mathrm{Ag} \mid \mathrm{AgCl}$ served as the reference, and a platinum plate was used as a counter electrode. A $0.1 \mathrm{M}$ solution of tetra butyl ammonium hexafluorophosphate in dichloromethane was used as the supporting electrolyte. A scan rate of $1 \mathrm{mV} / \mathrm{s}$ was used at room temperature. The inset represents a closeup of the current peaks at $-1.5,-1.75$, and $-1.9 \mathrm{~V}$. (b) Calculated electrochemical potentials for energy levels of $3 \mathrm{~nm} C \mathrm{C} S \mathrm{Se}$ QDs by assuming the conduction band (first excited state) potential as -1.75 V. (c) Discrete energy levels of $3 \mathrm{~nm}$ CdSe QDs. ${ }^{13,28} \mathrm{Up}$ and down arrows are connecting optically allowed transitions for absorption and emission. At the bottom, measured absorption and emission of the 3 $\mathrm{nm}$ CdSe QDs are also represented. (d) Energy levels of the conduction band and valence band of $\mathrm{TiO}_{2}$ and FTO.

QDs and the applied potentials. By identifying the electrochemical potential for the $\mathrm{CB}$ minimum (first excited state) of the QDs $U_{1 S_{e}}$ (in $\mathrm{V}$ ), the electrochemical potential for the valence band energy levels $U_{\mathrm{h}}$ can be expressed as ${ }^{13}$

$$
U_{\mathrm{h}}=U_{1 \mathrm{~S}_{\mathrm{e}}}+\Delta E_{\mathrm{opt}} / e+1.8 e /\left(4 \pi \varepsilon_{\mathrm{r}}^{\infty} \varepsilon_{0} r\right)
$$

where $\Delta E_{\text {opt }}$ is the energy of the optical transition from the corresponding energy level in the valence band to $1 S_{e}, e$ is the elementary charge, $\varepsilon_{\mathrm{r}}^{\infty}$ is the medium's relative permittivity at high frequencies, $\varepsilon_{0}$ is the vacuum permittivity, and $r$ is the radius of the QDs. The approximate electrochemical potentials for the energy levels obtained from the CV and the discrete energy levels of $3 \mathrm{~nm}$ CdSe QDs with their corresponding optical transitions are depicted in Figure 1b,c. ${ }^{13,14,26}$

In $\mathrm{CV}$, Figure $1 \mathrm{a}$, with the increasing negative bias, we see a steady raise of the current due to faradic processes. ${ }^{24}$ At -1.5 $\mathrm{V}$, a clear peak emerges since the Fermi level reaches the conduction band of $\mathrm{TiO}_{2}$ and, consequently, additional electrons can be injected into the $\mathrm{CB}$ of $\mathrm{TiO}_{2}$. At $-1.75 \mathrm{~V}$, another peak indicates the electrochemical potential where electrons can be transferred from $\mathrm{TiO}_{2}$ to the $1 \mathrm{~S}_{\mathrm{e}}$ level in the QDs. The next peak at $-1.9 \mathrm{~V}$ is at too low a potential to correspond to the next energy level, $1 \mathrm{P}_{\mathrm{e}}$, as illustrated in Figure $1 \mathrm{~b}, \mathrm{c}^{29}$ Accordingly, we interpret the peak as further charging of the QDs since the $1 S_{\mathrm{e}}$ level can simultaneously accommodate two electrons. Due to the Coulomb repulsion, it is expected that addition of the second electron requires more energy, which corresponds to a higher negative potential in electrochemistry. Since the features between -1.5 and $-1.75 \mathrm{~V}$ are a combination of those of the QDs and $\mathrm{TiO}_{2}-$ FTO, exact description of them solely based on the $\mathrm{CV}$ 
measurement is not straightforward. In addition, as one can see from the $\mathrm{CV}$ measurement in Figure $1 \mathrm{a}$ and as reported previously in the literature, the electrochemical measurements on QDs have an irreversible character. ${ }^{14,30,31}$ This indicates that the charges injected into the QDs do not fully return on the time scale of the electrochemical cycle when the potential is reversed. ${ }^{30}$ Previous studies have shown that charging can induce surface modifications, which lead to the formation of traps in QDs without inducing significant changes in absorption and photoluminescence. ${ }^{22}$ It has also been shown that it is possible to charge QDs without reducing the surface using a passivating ligand or by building core-shell structures to eliminate such surface species. ${ }^{21,22,32}$ For precise scrutiny of the effect of external bias on the QD assembly, as the main goal of this study, a combination of electrochemistry with spectroscopy $^{33}$ is used. Changes of the absorption spectra due to the state filling can further identify the energy levels that are involved in charging. ${ }^{13,34}$

\section{SPECTROELECTROCHEMISTRY}

The spectroelectrochemistry ${ }^{35}$ measurements were carried out by following the absorption spectra at different negative potentials during the $\mathrm{CV}$ scan. The initial spectrum was taken at the open-circuit potential (OCP) corresponding to $-0.25 \mathrm{~V}$ vs $\mathrm{Ag} \mid \mathrm{AgCl}$, and the potential-dependent spectral changes were obtained by subtracting this initial spectrum from the measured absorption spectra at each potential as represented in Figure 2.

The absorption spectra show two clear bands at around 550 $\mathrm{nm}$ for $1 \mathrm{~S}_{3 / 2}-1 \mathrm{~S}_{\mathrm{e}}$ and $2 \mathrm{~S}_{3 / 2}-1 \mathrm{~S}_{\mathrm{e}}$ transitions and at $450 \mathrm{~nm}$ for the $1 S_{1 / 2}-1 S_{e}$ transition. At all negative potentials, an increasing featureless background from 600 to $1000 \mathrm{~nm}$

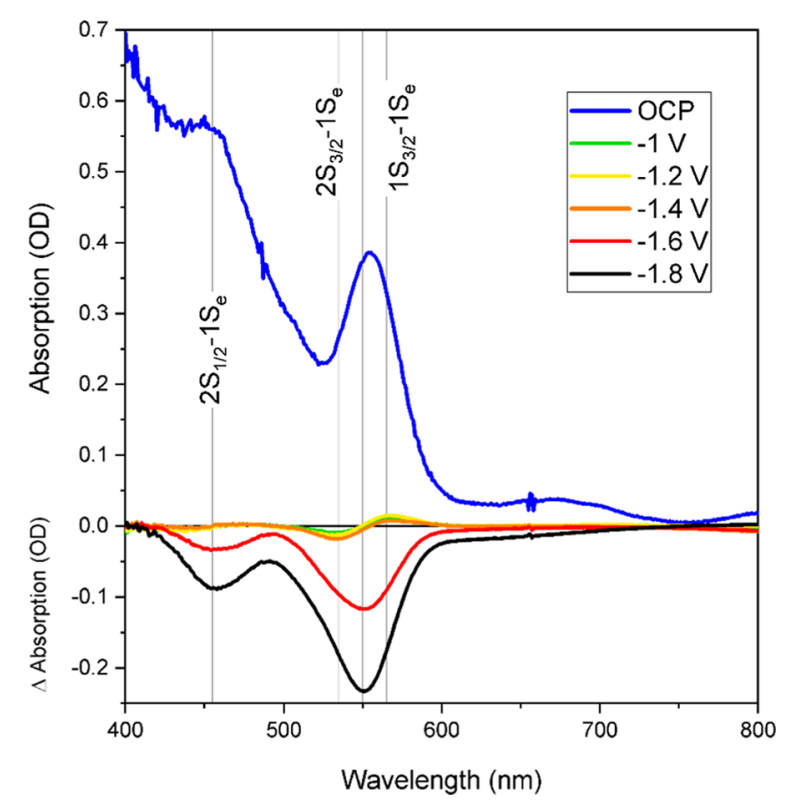

Figure 2. Spectroelectrochemical steady state absorption changes of 3 nm CdSe QDs in $\mathrm{TiO}_{2} /$ FTO. Upper part: steady state absorption of 3 $\mathrm{nm} \mathrm{CdSe}$ QDs in $\mathrm{TiO}_{2} /$ FTO where no potential is applied (OCP = $-0.25 \mathrm{~V}$ vs $\mathrm{Ag} \mid \mathrm{AgCl})$. Lower part: potential-dependent difference spectra obtained by subtraction of the OCP spectrum from the measured spectra. All potentials are measured vs the $\mathrm{Ag} \mid \mathrm{AgCl}$ pseudoreference, and a platinum plate is used as a counter electrode. A $0.1 \mathrm{M}$ solution of tetra butyl ammonium hexafluorophosphate in dichloromethane is used as the supporting electrolyte.
(Figure S5) is due to absorption of the charges in $\mathrm{TiO}_{2}{ }^{36}$ Until the potential reaches $-1.5 \mathrm{~V}$, the Fermi level in the $\mathrm{TiO}_{2}-\mathrm{FTO}$ layer is lower than the QD conduction band; hence, the induced absorption changes at 535 and $565 \mathrm{~nm}$ are due to the Stark shift. ${ }^{37}$ At $-1.6 \mathrm{~V}$ and a higher negative bias, a strong bleach of the transitions at 550 and $450 \mathrm{~nm}$ shows that the electrons fill the QD $1 S_{\mathrm{e}}$ state, and consequently, the absorption of all transitions to this level becomes partially bleached. At $-1.8 \mathrm{~V}$, the bleach amplitude becomes 2 times larger. These observations provide strong evidence that at -1.6 $\mathrm{V}$ the $\mathrm{QDs}$ are charged by one and at $-1.8 \mathrm{~V}$ by two electrons, which fill the doubly degenerate $1 S_{e}$ state. These results demonstrate that spectroelectrochemistry provides a convenient direct manipulation tool for a controlled charge injection to the QDs. In the following, we will use this method to prepare negatively charged QDs for the transient absorption measurements. We also point out that we are well aware of the limitations of the effective mass theory description and in reality the colloidal QDs have much more energy levels shown by atomistic calculations. ${ }^{38}$ Such a quasi-continuum view is important for understanding the excited electron relaxations in QDs, as discussed in the next section. However, the main spectral features of these two approaches are in good agreement, justifying our interpretation of electrochemistry in terms of $k \cdot p$ effective mass theory and state filling. It is worth mentioning that even at $-1.8 \mathrm{~V}$ the band edge transition is not fully bleached. This may be due to a subset of QDs with incomplete charging. This may also be the result of the size distribution of the QDs as the smaller dots are less likely to become fully charged. This means that in reality at $-1.8 \mathrm{~V}$ there is a mixture of states, which is making precise distinction of the processes in the following analyses complicated and thus the proposed models need to be considered as simplification of the full complexity of the real situation.

\section{TRANSIENT ABSORPTION SPECTROSCOPY}

Combination of spectroelectrochemistry with time-resolved femtosecond TA spectroscopy allows in situ probing of the excited state dynamics in well-controlled charged QDs. A detailed description of the TA measurements is given in the Supporting Information. Excitation at $400 \mathrm{~nm}$ was used to populate higher-energy levels $\left(2 \mathrm{P}_{1 / 2}-1 \mathrm{P}_{\mathrm{e}}\right.$ transition $)$ so that the absorption changes under different electrochemical potentials do not affect the excitation conditions. The initial hot electron relaxation provides valuable information about the details of the carrier cooling process. Decay-associated spectra (DAS) were obtained from global fitting of the TA data and used for mapping of the population and depopulation pathways of the excited states. The DAS spectra without normalization are presented in the Supporting Information. The TA measurements at OCP $(-0.25 \mathrm{~V}$ vs $\mathrm{AglAgCl})$ are taken as reference. In the following, the TA features are discussed in a consecutive order.

4.1. OCP and Negative Bias. Relaxation of the initially excited hot electrons and holes occurs in a sub-ps time scale. ${ }^{39}$ All DAS components of TA consist of a negative peak at 550 $\mathrm{nm}$ corresponding to a decay of the bleach signal related to the almost instantaneous state filling of $1 S_{3 / 2}-1 S_{e}$ and $2 S_{3 / 2}-1 S_{e}$ transitions. Since at OCP, after the laser excitation, only one electron and hole are present in QDs, electron-electron scattering cannot take place; hence, the relaxation is induced only by electron-phonon scattering. Since the phonon frequencies are significantly smaller than the level spacing in 
the effective mass description, the hot carrier cooling corresponds to relaxation through a quasi-continuum of the energy levels. ${ }^{38}$ The small positive band at $600 \mathrm{~nm}$ in the 500 fs DAS is the excitation-induced spectral shift and also reflects the hot carrier arrival to the band edge. ${ }^{40,41}$ It is followed by two slower processes corresponding to the initial electron injection ( 9 ps) to a charge transfer state where the electron still interacts with the hole and the full charge separation via electron diffusion to the bulk of $\mathrm{TiO}_{2}$ (95 ps). ${ }^{42}$ The broad positive signal at longer wavelengths is attributed to the excited state absorption (ESA) of free electrons in the $\mathrm{CB}$ of $\mathrm{TiO}_{2}{ }^{36}$ Eventually, the photogenerated charges are recovered within a time scale that is longer than our experimental limit noted as $>10 \mathrm{~ns}$ in all cartoon illustrations of the decay processes in Figure 3.

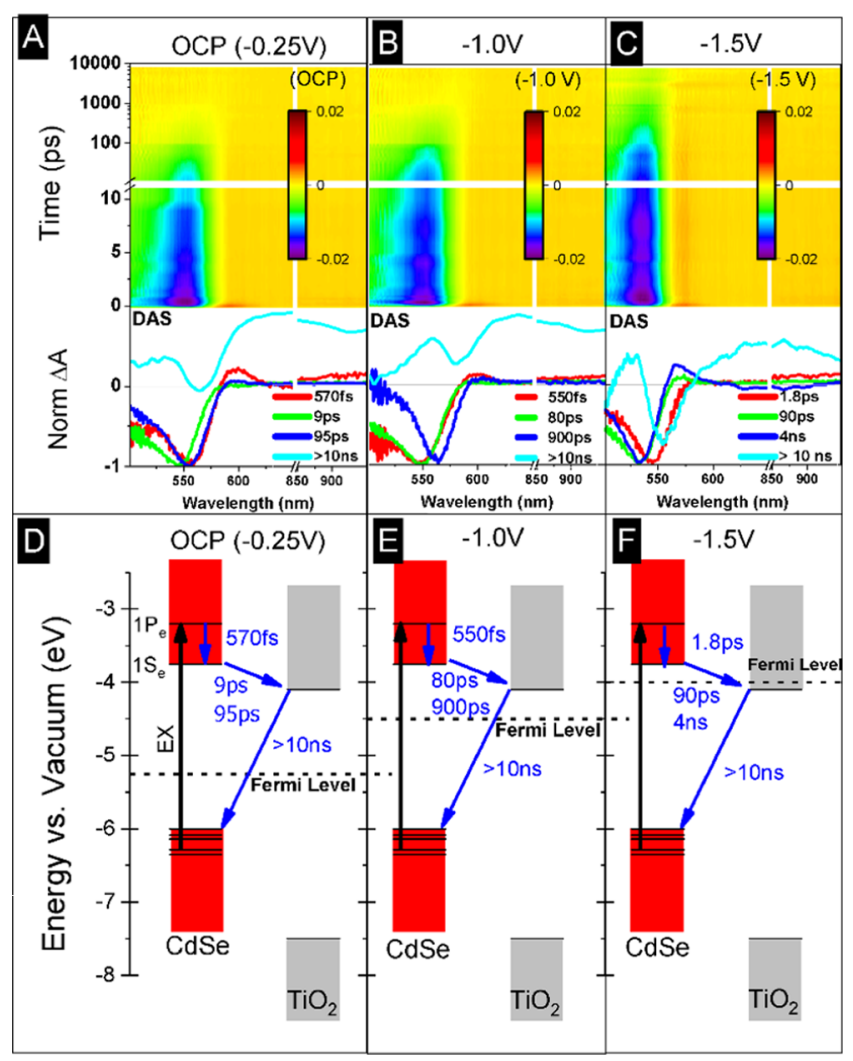

Figure 3. (A-C) Summary of TA measurements and their corresponding fitted exponents with normalized decay-associated spectra (DAS) of the $3 \mathrm{~nm} \mathrm{CdSe} \mathrm{QD} \mathrm{assembly} \mathrm{on} \mathrm{TiO}_{2}$ at OCP, -1 , and $-1.5 \mathrm{~V}$ applied bias vs $\mathrm{Ag} \mid \mathrm{AgCl}$, respectively. The corresponding unnormalized DAS component is shown in the Supporting Information. (D-F) Cartoon depiction of electron transitions at the given potentials. Black arrows indicate laser excitation connecting corresponding energy levels for photogenerated charges, and blue down arrows are used to show electron decaying pathways and recombination mechanisms.

4.2. Single and Double Charged QDs. When an external voltage is applied to the photoanode, at potentials up to -1.5 $\mathrm{V}$, no electrons are electrochemically injected into the QDs, but the excess electrons in $\mathrm{TiO}_{2}$-FTO do affect the excited state dynamics in the QDs as well as charge transfer to $\mathrm{TiO}_{2}$ due to long-range Coulomb interactions in QDs. ${ }^{43,44}$ At -1.0 $\mathrm{V}$, the Fermi level is still below the $\mathrm{CB}$ of $\mathrm{TiO}_{2}$ and the electrons can only enter the $\mathrm{TiO}_{2}$ shallow traps. ${ }^{45}$ The time scale of the hot electron relaxation is not influenced by this, while the photoexcited electron injection from the QDs to $\mathrm{TiO}_{2}$ and the following diffusion of the injected electron away from the $\mathrm{QD}$ vicinity into the bulk of $\mathrm{TiO}_{2}$ are both significantly slowed down from 9 to 80 ps and from 95 to $900 \mathrm{ps}$, respectively. At $-1.5 \mathrm{~V}$, when electrons fill the lowest levels of the $\mathrm{TiO}_{2}$ conduction band, the charge separation process is further slowed down up to ns. The fast component that was earlier related to the hot carrier cooling has also become significantly slower, representing a combination of the cooling and some other slower process.

When the applied negative bias reaches $-1.75 \mathrm{~V}$, the Fermi level of the $\mathrm{TiO}_{2}$-FTO layer is shifted higher than the QD $1 S_{\mathrm{e}}$ level, which becomes populated by one electron. Excitation of such charged QDs creates trions. The hot electron relaxation in the trion is shortened to $370 \mathrm{fs}$ due to the electron-electron scattering, which is now possible because of the extra electron. ${ }^{41}$ Since at $-1.75 \mathrm{~V}$, the electron density in the $\mathrm{CB}$ of the $\mathrm{TiO}_{2}$ is high, the electron injection from $\mathrm{QDs}$ to $\mathrm{TiO}_{2}$ is very unlikely to occur. The 5.8 ps component is interpreted as a combined effect of Coulombic repulsion between the electrons and polaron formation. ${ }^{40}$ The repulsion is likely to push the extra electron to the surface of the QDs. Such a new charge distribution is followed by nuclear rearrangement, forming a polaron and thereby stabilizing the charge distribution. $^{46}$ Observation of a strong Stokes shift of the fluorescence in electrochemically single and double charged CdSe QDs gives strong evidence for polaron formation and for the related nuclear rearrangement. ${ }^{20}$ The following 510 ps component is assigned to negative trion Auger recombination (see Figure 4A,C), which is in good agreement with the previous reports. ${ }^{15,18}$

After the Auger recombination, the QDs return back to the initial unexcited charged state, but the DAS analysis still shows a 12 ns component (10\% of the TA signal, Table S2) with a clear ground state bleach at the main absorption band. This minor long component corresponds to the QDs that have remained uncharged.

At $-2 \mathrm{~V}$, since QDs become double charged, the corresponding excited states are called tetrons, with three electrons and a hole. ${ }^{47}$ The hot electron relaxation (380 fs) has about the same time scale as in the case of the negative trions. The relaxation due to polaron formation becomes longer since stronger electrostatic repulsion can lead to larger charge redistribution and the corresponding rearrangement of the QDs' lattice needs more time to reach the new minimumenergy level. As expected, the Auger process in the tetron is slightly shorter (415 ps) than in the trion.

\section{CONCLUSIONS}

In conclusion, spectroelectrochemistry is used to directly manipulate and monitor charging of the $\mathrm{QD}$ photoanode assembly in a well-controlled manner. Combination of spectroelectrochemistry with in situ TA provided a convenient tool for the study of the excited state dynamics of the QDs under different potentials mimicking realistic working conditions in an optoelectronic device. At low potentials, the electrons cannot reach the $\mathrm{CB}$ of the $\mathrm{TiO}_{2}$ but populate the shallow traps, which appear as a constant featureless background over the whole visible spectral range. Next, the electrons start filling the $\mathrm{CB}$ of $\mathrm{TiO}_{2}$ without entering the QDs and the photoinduced electron transfer from the $\mathrm{QDs}$ to $\mathrm{TiO}_{2}$ is significantly slowed down. At even higher negative 


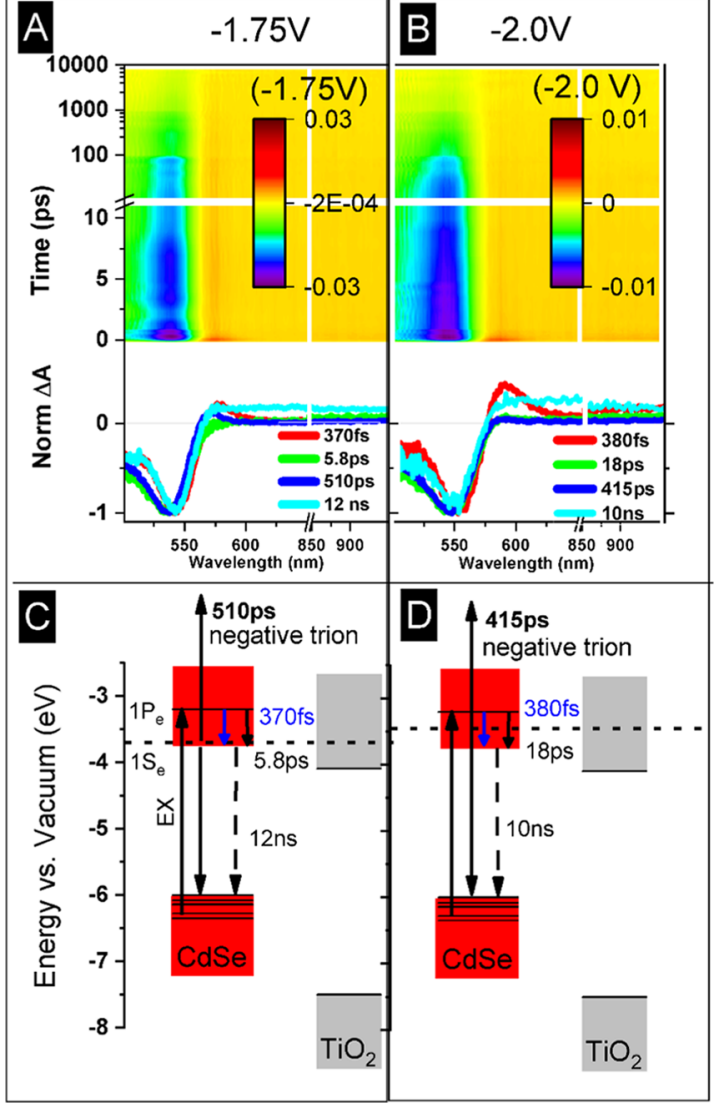

Figure 4. (A, B) Summary of TA measurement and the corresponding fitted exponents with decay-associated spectra for -1.75 and $-2.0 \mathrm{~V}$, respectively. The potentials are in respect to the $\mathrm{Ag} / \mathrm{AgCl}$ reference electrode. (C, D) Cartoon depiction of electron transitions, where black up arrows indicate laser excitation connecting corresponding energy levels for photogenerated charges and blue down arrows are used to show electron decaying pathways and recombination mechanisms. The Auger process is illustrated by double-head arrows to reflect the simultaneous recombination of electron-hole and excitation of an electron.

potentials, -1.75 and $-2.0 \mathrm{~V}$, spectroelectrochemistry confirmed that the QDs are charged by one and two electrons, respectively. Now the hot electron cooling process becomes faster due to the electron-electron scattering. In the charged QDs, a process in the picosecond time scale occurs, which is attributed to polaron formation. In addition, Auger relaxation with a 500 ps time constant is observed in our TA measurements. The Auger process becomes faster in the case of double charging, in tetrons. Thereby, we provide a thorough in situ spectroscopic investigation of QDs at operational conditions of devices, which depend on charge separation or electrocharging of the particles.

The work can lead to a novel photophysical view on the design of QDs in optoelectronic devices. Our exploration of the effect of slight variation of charging that causes diminution of charge extraction provides understanding of the photophysical ground for efficiency limits of the QD devices.

\section{ASSOCIATED CONTENT}

SI Supporting Information

The Supporting Information is available free of charge at https://pubs.acs.org/doi/10.1021/acsaem.0c02478.
Materials and chemicals; QD synthesis; film preparation; electrochemical measurement; spectroelectrochemistry; and transient absorption spectroscopy (PDF)

\section{AUTHOR INFORMATION}

\section{Corresponding Author}

Tönu Pullerits - Chemical Physics and NanoLund, Department of Chemistry, Lund University, 22100 Lund, Sweden; (1) orcid.org/0000-0003-1428-5564; Phone: +46 46 22281310; Email: tonu.pullerits@chemphys.lu.se; Fax: +46 462224119

\section{Authors}

Alireza Honarfar - Chemical Physics and NanoLund, Department of Chemistry, Lund University, 22100 Lund, Sweden; 이이.org/0000-0002-1852-3059

Hassan Mourad - Chemical Physics and NanoLund, Department of Chemistry, Lund University, 22100 Lund, Sweden

Weihua Lin - Chemical Physics and NanoLund, Department of Chemistry, Lund University, 22100 Lund, Sweden

Alexey Polukeev - Centre for Analysis and Synthesis, Department of Chemistry, Lund University, 22100 Lund, Sweden

Ahibur Rahaman - Chemical Physics and NanoLund, Department of Chemistry, Lund University, 22100 Lund, Sweden

Mohamed Abdellah - Chemical Physics and NanoLund, Department of Chemistry, Lund University, 22100 Lund, Sweden; Department of Chemistry, Qena Faculty of Science, South Valley University, 83523 Qena, Egypt

Pavel Chábera - Chemical Physics and NanoLund, Department of Chemistry, Lund University, 22100 Lund, Sweden; 이이이.org/0000-0002-0531-5138

Galina Pankratova - DTU Nanolab, National Centre for Nano Fabrication and Characterization, Technical University of Denmark, 2800 Kongens Lyngby, Denmark; Department of Biochemistry and Structural Biology, Lund University, 22100 Lund, Sweden

Lo Gorton - Department of Biochemistry and Structural Biology, Lund University, 22100 Lund, Sweden; (1) orcid.org/0000-0002-7278-0478

Kaibo Zheng - Chemical Physics and NanoLund, Department of Chemistry, Lund University, 22100 Lund, Sweden; Department of Chemistry, Technical University of Denmark, 2800 Kongens Lyngby, Denmark; 이이이.org/0000-00027236-1070

Complete contact information is available at: https://pubs.acs.org/10.1021/acsaem.0c02478

\section{Author Contributions}

A.H. and H.M. contributed equally. The manuscript was written through contributions of all authors. All authors have given approval to the final version of the manuscript.

\section{Funding}

K.Z. acknowledges financial support from the Independent Research Fund Denmark, Sapere Aude Starting Grant (No. 7026-00037A), and the Swedish Research Council VR Starting Grant (No. 2017-05337). W.L. thanks financial support by the China Scholarship Council (CSC) under Grant No. 201806460021 


\section{Notes}

The authors declare no competing financial interest.

\section{ACKNOWLEDGMENTS}

The authors acknowledge funding by the Swedish Research Council, Swedish Energy Agency, KAW Foundation, and NanoLund. A.H. thanks Prof. Ebbe Nordlander, Prof. Ola F. Wendt, and Dr. Carlito S. Ponseca for their scientific support.

\section{ABBREVIATIONS}

QD, quantum dot

$\mathrm{CB}$, conduction band

$\mathrm{CV}$, cyclic voltammogram

FTO, fluorine-doped tin oxide

OCP, open-circuit potential

TA, transient absorption spectroscopy

DAS, decay-associated spectra

ESA, excited state absorption

\section{REFERENCES}

(1) Klimov, V. I. Nanocrystal Quantum Dots, 2nd ed.; CRC Press, 2017.

(2) Efros, A. L.; Rosen, M.; Kuno, M.; Nirmal, M.; Norris, D. J.; Bawendi, M. Band-Edge Exciton in Quantum Dots of Semiconductors with a Degenerate Valence Band: Dark and Bright Exciton States. Phys. Rev. B 1996, 54, No. 4843.

(3) Zheng, K.; Zídek, K.; Abdellah, M.; Zhang, W.; Chábera, P.; Lenngren, N.; Yartsev, A.; Pullerits, T. Ultrafast Charge Transfer from CdSe Quantum Dots to P-Type NiO: Hole Injection vs Hole Trapping. J. Phys. Chem. C 2014, 118, 18462-18471.

(4) Chuang, C.-H. M.; Brown, P. R.; Bulović, V.; Bawendi, M. G. Improved Performance and Stability in Quantum Dot Solar Cells through Band Alignment Engineering. Nat. Mater. 2014, 13, 796801.

(5) Lim, J.; Park, Y.-S.; Klimov, V. I. Optical Gain in Colloidal Quantum Dots Achieved with Direct-Current Electrical Pumping. Nat. Mater. 2018, 17, 42-49.

(6) Grimaldi, G.; Geuchies, J. J.; van der Stam, W.; du Fossé, I.; Brynjarsson, B.; Kirkwood, N.; Kinge, S.; Siebbeles, L. D. A.; Houtepen, A. J. Spectroscopic Evidence for the Contribution of Holes to the Bleach of Cd-Chalcogenide Quantum Dots. Nano Lett. 2019, 19, 3002-3010.

(7) Kamat, P. V. Quantum Dot Solar Cells. The Next Big Thing in Photovoltaics. J. Phys. Chem. Lett. 2013, 4, 908-918.

(8) Tvrdy, K.; Frantsuzov, P. A.; Kamat, P. V. Photoinduced Electron Transfer from Semiconductor Quantum Dots to Metal Oxide Nanoparticles. Proc. Natl. Acad. Sci. U.S.A. 2011, 108, 29-34.

(9) Ž́idek, K.; Zheng, K.; Abdellah, M.; Lenngren, N.; Chábera, P.; Pullerits, T. Ultrafast Dynamics of Multiple Exciton Harvesting in the $\mathrm{CdSe}-\mathrm{ZnO}$ System: Electron Injection versus Auger Recombination. Nano Lett. 2012, 12, 6393-6399.

(10) Brennan, K. F. The Physics of Semiconductors: With Applications to Optoelectronic Devices; Cambridge University Press: Cambridge, 1999.

(11) Zang, H.; Hsiao, Y.-C.; Hu, B. Surface-Charge Accumulation Effects on Open-Circuit Voltage in Organic Solar Cells Based on Photoinduced Impedance Analysis. Phys. Chem. Chem. Phys. 2014, 16, 4971-4976.

(12) Boehme, S. C.; Walvis, T. A.; Infante, I.; Grozema, F. C.; Vanmaekelbergh, D.; Siebbeles, L. D. A.; Houtepen, A. J. Electrochemical Control over Photoinduced Electron Transfer and Trapping in CdSe-CdTe Quantum-Dot Solids. ACS Nano 2014, 8, 7067-7077.

(13) Spittel, D.; Poppe, J.; Meerbach, C.; Ziegler, C.; Hickey, S. G.; Eychmüller, A. Absolute Energy Level Positions in CdSe Nanostructures from Potential-Modulated Absorption Spectroscopy (EMAS). ACS Nano 2017, 11, 12174-12184.
(14) Ingole, P. P. A Consolidated Account of Electrochemical Determination of Band Structure Parameters in II-VI Semiconductor Quantum Dots: A Tutorial Review. Phys. Chem. Chem. Phys. 2019, 21, $4695-4716$.

(15) Jha, P. P.; Guyot-Sionnest, P. Trion Decay in Colloidal Quantum Dots. ACS Nano 2009, 3, 1011-1015.

(16) Singh, R.; Liu, W.; Lim, J.; Robel, I.; Klimov, V. I. Hot-Electron Dynamics in Quantum Dots Manipulated by Spin-Exchange Auger Interactions. Nat. Nanotechnol. 2019, 14, 1035-1041.

(17) Wu, K.; Lim, J.; Klimov, V. I. Superposition Principle in Auger Recombination of Charged and Neutral Multicarrier States in Semiconductor Quantum Dots. ACS Nano 2017, 11, 8437-8447.

(18) Hou, X.; Kang, J.; Qin, H.; Chen, X.; Ma, J.; Zhou, J.; Chen, L.; Wang, L.; Wang, L.-W.; Peng, X. Engineering Auger Recombination in Colloidal Quantum Dots via Dielectric Screening. Nat. Commun. 2019, 10, No. 1750.

(19) Abdellah, M.; Žídek, K.; Zheng, K.; Chábera, P.; Messing, M. E.; Pullerits, T. Balancing Electron Transfer and Surface Passivation in Gradient CdSe/ZnS Core-Shell Quantum Dots Attached to ZnO. J. Phys. Chem. Lett. 2013, 4, 1760-1765.

(20) Wang, C.; Wehrenberg, B. L.; Woo, C. Y.; Guyot-Sionnest, P. Light Emission and Amplification in Charged CdSe Quantum Dots. J. Phys. Chem. B 2004, 108, 9027-9031.

(21) Li, B.; Lu, M.; Liu, W.; Zhu, X.; He, X.; Yang, Y.; Yang, Q. Reversible Electrochemical Control over Photoexcited Luminescence of Core/Shell CdSe/ZnS Quantum Dot Film. Nanoscale Res. Lett. 2017, 12, No. 626.

(22) du Fossé, I.; ten Brinck, S.; Infante, I.; Houtepen, A. J. Role of Surface Reduction in the Formation of Traps in N-Doped II-VI Semiconductor Nanocrystals: How to Charge without Reducing the Surface. Chem. Mater. 2019, 31, 4575-4583.

(23) Geuchies, J. J.; Brynjarsson, B.; Grimaldi, G.; Fossé, I. D.; Dijkhuizen, R.; Koel, M.; Gudjonsdottir, S.; Stam, W.; van der Evers, W. H.; Geiregat, P.; Hens, Z.; Houtepen, A. J. Quantitative Electrochemical Control over Optical Gain in Colloidal QuantumDot and Quantum-Well Solids. In Physical Chemistry of Semiconductor Materials and Interfaces XIX; International Society for Optics and Photonics, 2020; Vol. 11464, p 114640R.

(24) Bard, A. J.; Faulkner, L. R. Electrochemical Methods: Fundamentals and Applications, 2nd ed.; John Wiley, 2008.

(25) Elgrishi, N.; Rountree, K. J.; McCarthy, B. D.; Rountree, E. S.; Eisenhart, T. T.; Dempsey, J. L. A Practical Beginner's Guide to Cyclic Voltammetry. J. Chem. Educ. 2018, 95, 197-206.

(26) Kucur, E.; Riegler, J.; Urban, G. A.; Nann, T. Determination of Quantum Confinement in CdSe Nanocrystals by Cyclic Voltammetry. J. Chem. Phys. 2003, 119, 2333-2337.

(27) Margaritondo, G. Electronic Structure of Semiconductor Heterojunctions; Springer: Netherlands, 1988.

(28) Gaponenko, S. V.; Demir, H. V. Applied Nanophotonics; Cambridge University Press: Cambridge, 2018.

(29) Yu, D.; Wang, C.; Guyot-Sionnest, P. N-Type Conducting CdSe Nanocrystal Solids. Science 2003, 300, 1277-1280.

(30) Gudjonsdottir, S.; van der Stam, W.; Koopman, C.; Kwakkenbos, B.; Evers, W. H.; Houtepen, A. J. On the Stability of Permanent Electrochemical Doping of Quantum Dot, Fullerene, and Conductive Polymer Films in Frozen Electrolytes for Use in Semiconductor Devices. ACS Appl. Nano Mater. 2019, 2, 4900-4909.

(31) Inamdar, S. N.; Ingole, P. P.; Haram, S. K. Determination of Band Structure Parameters and the Quasi-Particle Gap of CdSe Quantum Dots by Cyclic Voltammetry. ChemPhysChem 2008, 9, 2574-2579.

(32) van der Stam, W.; Grimaldi, G.; Geuchies, J. J.; Gudjonsdottir, S.; van Uffelen, P. T.; van Overeem, M.; Brynjarsson, B.; Kirkwood, N.; Houtepen, A. J. Electrochemical Modulation of the Photophysics of Surface-Localized Trap States in Core/Shell/(Shell) Quantum Dot Films. Chem. Mater. 2019, 31, 8484-8493.

(33) Lozeman, J. J. A.; Führer, P.; Olthuis, W.; Odijk, M. Spectroelectrochemistry, the Future of Visualizing Electrode 
Processes by Hyphenating Electrochemistry with Spectroscopic Techniques. Analyst 2020, 145, 2482-2509.

(34) Wang, C.; Shim, M.; Guyot-Sionnest, P. Electrochromic Nanocrystal Quantum Dots. Science 2001, 291, 2390-2392.

(35) Kaim, W.; Klein, A. Spectroelectrochemistry; RSC Publishing, 2008.

(36) Maity, P.; Mohammed, O. F.; Katsiev, K.; Idriss, H. Study of the Bulk Charge Carrier Dynamics in Anatase and Rutile TiO2 Single Crystals by Femtosecond Time-Resolved Spectroscopy. J. Phys. Chem. C 2018, 122, 8925-8932.

(37) Colvin, V. L.; Cunningham, K. L.; Alivisatos, A. P. Electric Field Modulation Studies of Optical Absorption in CdSe Nanocrystals: Dipolar Character of the Excited State. J. Chem. Phys. 1994, 101, 7122-7138.

(38) Prezhdo, O. V. Multiple Excitons and the Electron-Phonon Bottleneck in Semiconductor Quantum Dots: An Ab Initio Perspective. Chem. Phys. Lett. 2008, 460, 1-9.

(39) Klimov, V. I.; McBranch, D. W. Femtosecond 1P-to-1S Electron Relaxation in Strongly Confined Semiconductor Nanocrystals. Phys. Rev. Lett. 1998, 80, No. 4028.

(40) Karki, K. J.; Widom, J. R.; Seibt, J.; Moody, I.; Lonergan, M. C.; Pullerits, T.; Marcus, A. H. Coherent Two-Dimensional Photocurrent Spectroscopy in a PbS Quantum Dot Photocell. Nat. Commun. 2014, 5, No. 5869.

(41) Chen, J.; Messing, M. E.; Zheng, K.; Pullerits, T. CationDependent Hot Carrier Cooling in Halide Perovskite Nanocrystals. J. Am. Chem. Soc. 2019, 141, 3532-3540.

(42) Žídek, K.; Zheng, K.; Ponseca, C. S.; Messing, M. E.; Wallenberg, L. R.; Chábera, P.; Abdellah, M.; Sundström, V.; Pullerits, $\mathrm{T}$. Electron Transfer in Quantum-Dot-Sensitized $\mathrm{ZnO}$ Nanowires: Ultrafast Time-Resolved Absorption and Terahertz Study. J. Am. Chem. Soc. 2012, 134, 12110-12117.

(43) Leatherdale, C. A.; Kagan, C. R.; Morgan, N. Y.; Empedocles, S. A.; Kastner, M. A.; Bawendi, M. G. Photoconductivity in CdSe Quantum Dot Solids. Phys. Rev. B 2000, 62, No. 2669.

(44) Whan, C. B.; White, J.; Orlando, T. P. Full Capacitance Matrix of Coupled Quantum Dot Arrays: Static and Dynamical Effects. Appl. Phys. Lett. 1996, 68, 2996-2998.

(45) Antila, L. J.; Santomauro, F. G.; Hammarström, L.; Fernandes, D. L. A.; Sá, J. Hunting for the Elusive Shallow Traps in $\mathrm{TiO}_{2}$ Anatase. Chem. Commun. 2015, 51, 10914-10916.

(46) Zheng, K.; Abdellah, M.; Zhu, Q.; Kong, Q.; Jennings, G.; Kurtz, C. A.; Messing, M. E.; Niu, Y.; Gosztola, D. J.; Al-Marri, M. J.; Zhang, X.; Pullerits, T.; Canton, S. E. Direct Experimental Evidence for Photoinduced Strong-Coupling Polarons in Organolead Halide Perovskite Nanoparticles. J. Phys. Chem. Lett. 2016, 7, 4535-4539.

(47) Hartstein, K. H.; Erickson, C. S.; Tsui, E. Y.; Marchioro, A.; Gamelin, D. R. Electron Stability and Negative-Tetron Luminescence in Free-Standing Colloidal n-Type CdSe/CdS Quantum Dots. ACS Nano 2017, 11, 10430-10438. 